\title{
Bézier control points method to solve constrained quadratic optimal control of time varying linear systems
}

\author{
F. GHOMANJANI, M.H. FARAHI and M. GACHPAZAN \\ Department of Applied Mathematics, Faculty of Mathematical Sciences \\ Ferdowsi University of Mashhad, Mashhad, Iran \\ E-mail: gachpazan@ferdowsi.um.ac.ir
}

\begin{abstract}
A computational method based on Bézier control points is presented to solve optimal control problems governed by time varying linear dynamical systems subject to terminal state equality constraints and state inequality constraints. The method approximates each of the system state variables and each of the control variables by a Bézier curve of unknown control points. The new approximated problems converted to a quadratic programming problem which can be solved more easily than the original problem. Some examples are given to verify the efficiency and reliability of the proposed method.
\end{abstract}

\section{Mathematical subject classification: $49 \mathrm{~N} 10$.}

Key words: Bézier control points, constrained optimal control, linear time varying dynamical systems.

\section{Introduction}

In the numerical solution of differential equations, polynomial or piecewise polynomial functions are often used to represent the approximate solution [1]. Legendre and Chebyshev polynomials are used for solving optimal control problems (see [2], [3], [4] and [5]). Razzaghi and Yousefi [6] defined functions which they called Legendre wavelets for solving constrained optimal control problem. In particular, B-splines and Bezier curves have become popular tools for solving dynamical systems [7]. For both Bezier and B-spline representations, the curve 
or surface shape is outlined by a "control polygon" that is formed by connecting the control points. Since the control point structure captures important geometric features of the Bezier or B-spline shape, it is tempting to perform computations just based on the control points. This paper intends to investigate the use of the control points of Bezier representations for solving optimal control problems with inequality constrained. Since the use of B-splines is to cause the continuity of control curves, we propose to represent the approximate solution in Bezier curves for time varying optimal control problem with inequality constraints. The choice of the Bezier form rather than the B-spline form is due to the fact that the Bezier form is easier to symbolically carry out the operations of multiplication, composition and degree elevation than the B-spline form. We choose the sum of squares of the Bezier control points of the residual to be the measure quantity. Minimizing this quantity gives the approximate solution. Obviously, if the quantity is zero, then the residual function is also zero, which implies the solution is the exact solution. We call this approach the control-point-based method.

Consider the following time varying optimal control problem with pathwise state inequality constraints,

$$
\min \operatorname{Cost}=\frac{1}{2} \mathbf{x}\left(t_{f}\right)^{T} H\left(t_{f}\right) \mathbf{x}\left(t_{f}\right)+\int_{t_{0}}^{t_{f}}\left(\mathbf{x}^{T} P \mathbf{x}+\mathbf{u}^{T} Q \mathbf{u}+K \mathbf{x}+R \mathbf{u}\right) d t
$$

s.t.

$$
\begin{aligned}
& \dot{\mathbf{x}}(t)=A(t) \mathbf{x}(t)+B(t) \mathbf{u}(t)+F(t), \mathbf{x}\left(t_{0}\right)=\mathbf{x}_{0}, \\
& l_{i}\left(\mathbf{x}\left(t_{f}\right)\right)=0, \quad i \in E=\left\{1,2, \ldots, N_{1}\right\}, \\
& g_{h}\left(\mathbf{x}\left(t_{f}\right)\right) \leq 0, \quad h \in I=\left\{1,2, \ldots, N_{2}\right\}, \\
& q(t, \mathbf{x}(t)) \leq 0, \quad t \in\left[t_{0}, t_{f}\right],
\end{aligned}
$$

where

$$
\begin{gathered}
H(t)=\left[h_{i j}(t)\right]_{p \times p}, \quad P(t)=\left[p_{i j}(t)\right]_{p \times p}, \quad Q(t)=\left[q_{i j}(t)\right]_{m \times m}, \\
A(t)=\left[a_{i j}(t)\right]_{p \times p} \quad \text { and } \quad B(t)=\left[b_{i j}(t)\right]_{p \times m}
\end{gathered}
$$

are matrices functions and $K(t)=\left(k_{1}(t) \ldots k_{p}(t)\right), R(t)=\left(r_{1}(t) \ldots r_{m}(t)\right)$, $F(t)=\left(f_{1}(t) \ldots f_{p}(t)\right)^{T}$ are vectors functions, where the entries of mentioned matrices and polynomials in $\left[t_{0}, t_{f}\right]$. One may note when the entries of 
mentioned matrices are not polynomials in $\left[t_{0}, t_{f}\right]$, Taylor series is used for approximation. $\mathbf{x}(t)=\left(x_{1}(t) x_{2}(t) \ldots x_{p}(t)\right)^{T} \in R^{p}$ is $p \times 1$ trajectory state vector, $\mathbf{u}(t) \in \mathbb{U}=\left\{\left(u_{1}(t) u_{2}(t) \ldots u_{m}(t)\right)^{T}: b_{-}^{i}(t) \leq u_{i} \leq b_{+}^{i}(t), \quad 1 \leq i \leq\right.$ $m\} \subseteq R^{m}$ is $m \times 1$ control vector. The fixed finite terminal time $t_{f}$ is given, and $\mathbf{x}_{0}$ is the vector of initial conditions. In addition, $l_{i}: R^{p} \rightarrow R$ for $i \in E$, $g_{h}: R^{p} \rightarrow R$ for $h \in I, q:\left[t_{0}, t_{f}\right] \times R^{p} \rightarrow R$, and $b_{-}^{i}(t), b_{+}^{i}(t), 1 \leq i \leq m$ are given bounded functions.

The constrained optimal control of a linear system with a quadratic performance index has been of considerable concern and is well covered in many papers (see [6] and [8]). One of the methods to solve constrained optimal control problem (1), based on parameterizing the state/control variables, which convert the problem to a finite dimensional optimization problem, i.e. a mathematical programming problem (see [9], [10], [11]-[16]).

Analytical techniques developed in [5] are of benefit also in studying the convergence properties of related algorithms for solving optimal control problems, involving Chebyshev type functional constraints where, owing to the use of a variable step-size in integration or high order integration procedures, it is either not possible or inconvenient to base the analysis on an a priori discretization of the dynamic. The method used slack variables to convert the inequality constraints into equality constraints. This approach has an obvious disadvantage when applied to constrained optimal control of time varying linear problems because it converts the linear constraints into a nonlinear one (see [17]).

In this paper, we show a novel strategy by using the Bézier curves to find the approximate solution for (1). In this method, we divided the time interval, into $k$ subintervals and approximate the trajectory and control functions in each subinterval by Bézier curves. We have chosen the Bézier curves as piecewise polynomials of degree $n$, and determine Bézier curves on any subinterval by $n+1$ control points. By involving a least square optimization problem, one can find the control points, where the Bézier curves that approximate the action of control and trajectory, can be found as well (see [7] and [18]).

To show the effectiveness of this method the computational results of three examples are presented and compared with the results obtained in [5] and [17]. 


\section{Least square method}

Let $k$ be a chosen positive integer and $\left\{t_{0}<t_{1}<\cdots<t_{k}=t_{f}\right\}$ be an equidistance partition of $\left[t_{0}, t_{f}\right]$ with length $\tau$ and $S_{j}=\left[t_{j-1}, t_{j}\right]$ for $j=1,2, \ldots, k$. We define the following suboptimal control problems

$$
\min _{\operatorname{Cost}}=C_{j k}+\int_{t_{j-1}}^{t_{j}}\left(\mathbf{x}_{j}^{T} P \mathbf{x}_{j}+\mathbf{u}_{j}^{T} Q \mathbf{u}_{j}+K \mathbf{x}_{j}+R \mathbf{u}_{j}\right) d t
$$

s.t.

$$
\begin{aligned}
& \dot{\mathbf{x}}_{j}(t)=A(t) \mathbf{x}_{j}(t)+B(t) \mathbf{u}_{j}(t)+F(t), \mathbf{x}_{1}\left(t_{0}\right)=\mathbf{x}_{0}, \quad t \in S_{j}, \\
& l_{i}\left(\mathbf{x}_{k}\left(t_{f}\right)\right)=0, \quad i \in E \\
& g_{h}\left(\mathbf{x}_{k}\left(t_{f}\right)\right) \leq 0, \quad h \in I, \\
& q\left(t, \mathbf{x}_{j}(t)\right) \leq 0, \quad t \in S_{j}, j=1,2, \ldots, k
\end{aligned}
$$

where $C_{j k}=\frac{1}{2} \delta_{j k} \mathbf{x}_{k}^{T}\left(t_{f}\right) H\left(t_{f}\right) \mathbf{x}_{k}\left(t_{f}\right)$, and $\delta_{j k}$ is the Kronecker delta which has the value of unity when $j=k$ and otherwise is zero.

$$
\mathbf{x}_{j}(t)=\left(x_{1}^{j}(t) x_{2}^{j}(t) \ldots x_{p}^{j}(t)\right)^{T} \text { and } \mathbf{u}_{j}(t)=\left(u_{1}^{j}(t) u_{2}^{j}(t) \ldots u_{m}^{j}(t)\right)^{T}
$$

are respectively vectors of $\mathbf{x}(t)$ and $\mathbf{u}(t)$ when are considered in $S_{j}=\left[t_{j-1}, t_{j}\right]$.

Our strategy is using Bezier curves to approximate the solutions $\mathbf{x}_{j}(t)$ and $\mathbf{u}_{j}(t)$ by $\mathbf{v}_{j}(t)$ and $\mathbf{w}_{j}(t)$ respectively, where $\mathbf{v}_{j}(t)$ and $\mathbf{w}_{j}(t)$ are given below. Individual Bezier curves that are defined over the subintervals are joined together to form the Bezier spline curves. For $j=1,2, \ldots, k$, define the Bezier polynomials of degree $n$ that approximate the actions of $\mathbf{x}_{j}(t)$ and $\mathbf{u}_{j}(t)$ over the interval $S_{j}=\left[t_{j-1}, t_{j}\right]$ as follows:

$$
\begin{aligned}
& \mathbf{v}_{j}(t)=\sum_{r=0}^{n} \mathbf{a}_{r}^{j} B_{r, n}\left(\frac{t-t_{j-1}}{\tau}\right), \\
& \mathbf{w}_{j}(t)=\sum_{r=0}^{n} \mathbf{b}_{r}^{j} B_{r, n}\left(\frac{t-t_{j-1}}{\tau}\right),
\end{aligned}
$$

where

$$
B_{r, n}\left(\frac{t-t_{j-1}}{\tau}\right)=\left(\begin{array}{l}
n \\
r
\end{array}\right) \frac{1}{\tau^{n}}\left(t_{j}-t\right)^{n-r}\left(t-t_{j-1}\right)^{r},
$$


is the Bernstein polynomial of degree $n$ over the interval $\left[t_{j-1}, t_{j}\right], \mathbf{a}_{r}^{j}$ and $\mathbf{b}_{r}^{j}$ are respectively $p$ and $m$ ordered vectors from the control points. By substituting (3) in (2), one may define residual $R_{1, j}(t)$ and $R_{2, j}(t)$ for $t \in\left[t_{j-1}, t_{j}\right]$ as:

$$
\begin{aligned}
& R_{1, j}(t)=\dot{\mathbf{v}}_{j}(t)-A(t) \mathbf{v}_{j}(t)-B(t) \mathbf{w}_{j}(t)-F(t), \\
& R_{2, j}(t)=\mathbf{v}_{j}^{T}(t) P(t) \mathbf{v}_{j}(t)+\mathbf{w}_{j}^{T}(t) Q(t) \mathbf{w}_{j}(t)+K(t) \mathbf{v}_{j}(t)+R(t) \mathbf{w}_{j}(t) .
\end{aligned}
$$

Beside the boundary conditions on $\mathbf{v}(t)$, in nodes, there are also continuity constraints imposed on each successive pair of Bezier curves. Since the differential equation is of the first order, the continuity of $\mathbf{x}$ (or $\mathbf{v}$ ) and its first derivative gives

$$
\mathbf{v}_{j}^{(s)}\left(t_{j}\right)=\mathbf{v}_{j+1}^{(s)}\left(t_{j}\right), s=0,1, j=1,2, \ldots, k-1 .
$$

where $\mathbf{v}_{j}^{(s)}\left(t_{j}\right)$ is the $s$-th derivative of $\mathbf{v}_{j}(t)$ with respect to $t$ at $t=t_{j}$.

Thus the vector of control points $\mathbf{a}_{r}^{j}$ (for $r=0,1, n-1$ and $n$ ) must satisfy

$$
\begin{aligned}
& \mathbf{a}_{n}^{j}\left(t_{j}-t_{j-1}\right)^{n}=\mathbf{a}_{0}^{j+1}\left(t_{j+1}-t_{j}\right)^{n}, \\
& \left(\mathbf{a}_{n}^{j}-\mathbf{a}_{n-1}^{j}\right)\left(t_{j}-t_{j-1}\right)^{n-1}=\left(\mathbf{a}_{1}^{j+1}-\mathbf{a}_{0}^{j+1}\right)\left(t_{j+1}-t_{j}\right)^{n-1} .
\end{aligned}
$$

One may recall that $\mathbf{a}_{r}^{j}$ is an $p$ ordered vector. This approach is called the subdivision scheme (or $\tau$-refinement in the finite element literature), in the Section 3, we prove the convergence in the approximation with Bezier curves when $n$ tends to infinity.

Note 1: If we consider the $C^{1}$ continuity of $\mathbf{w}$, the following constraints will be added to constraints (5),

$$
\begin{aligned}
& \mathbf{b}_{n}^{j}\left(t_{j}-t_{j-1}\right)^{n}=\mathbf{b}_{0}^{j+1}\left(t_{j+1}-t_{j}\right)^{n}, \\
& \left(\mathbf{b}_{n}^{j}-\mathbf{b}_{n-1}^{j}\right)\left(t_{j}-t_{j-1}\right)^{n-1}=\left(\mathbf{b}_{1}^{j+1}-\mathbf{b}_{0}^{j+1}\right)\left(t_{j+1}-t_{j}\right)^{n-1},
\end{aligned}
$$

where the so-called $\mathbf{b}_{r}^{j}$ is an $m$ ordered vector.

Now, we define residual function in $S_{j}$ as follows

$$
R_{j}=\left(C_{j k}\right)^{2}+\int_{t_{j-1}}^{t_{j}}\left(M\left\|R_{1, j}(t)\right\|^{2}+\left(R_{2, j}(t)\right)^{2}\right) d t,
$$


where $\|$.$\| is the Euclidean norm (Recall that R_{1, j}(t)$ is a $p$ vector where $t \in S_{j}$ ) and $M$ is a sufficiently large penalty parameter. Our aim is to solve the following problem over $S=\bigcup_{j=1}^{k} S_{j}$,

$$
\begin{array}{ll} 
& \min \sum_{j=1}^{k} R_{j} \\
\text { s.t. } & \\
& l_{i}\left(\mathbf{v}_{k}\left(t_{k}\right)\right)=0, \quad i \in E, \\
& g_{h}\left(\mathbf{v}_{k}\left(t_{k}\right)\right) \leq 0, \quad h \in I, \\
& q\left(t, \mathbf{v}_{j}(t)\right) \leq 0, \quad t \in S_{j}, j=1,2, \ldots, k, \\
& \mathbf{v}_{j}^{(s)}\left(t_{j}\right)=\mathbf{v}_{j+1}^{(s)}\left(t_{j}\right), s=0,1, j=1,2, \ldots, k-1 .
\end{array}
$$

The mathematical programming problem (7) can be solved by many subroutines, we used Maple 12 to solve this optimization problem.

Note 2: If in time varying optimal control problem (1), $x\left(t_{f}\right)$ be unknown, then we set $C_{k k}=0$.

Note 3: To find good polynomial approximation, one needs to increase the degree of polynomial, in this article, we used sectional approximation, to find accurate results by low degree polynomials.

\section{Convergence analysis}

In this section, we analyze the convergence of the control-point-based method when applied to the following time varying optimal control problem:

$$
\begin{aligned}
\min I= & \frac{1}{2} x(1) H(1) x(1)+\int_{0}^{1}(x(t) P(t) x(t)+u(t) Q(t) u(t) \\
& +K(t) x(t)+R(t) u(t)) d t
\end{aligned}
$$

s.t.

$$
\begin{aligned}
& L(x(t), u(t), \dot{x}(t))=\dot{x}(t)-A(t) x(t)-B(t) u(t)=F(t), \quad t \in[0,1] \\
& x(0)=x_{0}=a, \quad x(1)=x_{f}=b, u(0)=u_{0}=a_{1} .
\end{aligned}
$$


where the state $x(t) \in R, u(t) \in R, a, b \in R, H(t), P(t), Q(t), K(t), R(t)$, $A(t), B(t)$ and $F(t)$ are polynomials in $[0,1]$.

Without loss of generality, we consider the interval $[0,1]$ instead of $\left[t_{0}, t_{f}\right]$, since one can change the variable $t$ with the new variable $z$ by $t=\left(t_{f}-t_{0}\right) z+t_{0}$ where $z \in[0,1]$.

Lemma 3.1. For a polynomial in Bezier form

$$
x(t)=\sum_{i=0}^{n_{1}} a_{i, n_{1}} B_{i, n_{1}}(t)
$$

we have

$$
\frac{\sum_{i=0}^{n_{1}} a_{i, n_{1}}^{2}}{n_{1}+1} \geq \frac{\sum_{i=0}^{n_{1}+1} a_{i, n_{1}+1}^{2}}{n_{1}+2} \geq \ldots \geq \frac{\sum_{i=0}^{n_{1}+m_{1}} a_{i, n_{1}+m_{1}}^{2}}{n_{1}+m_{1}+1},
$$

where $a_{i, n_{1}+m_{1}}$ is the Bezier coefficients of $x(t)$ after it is degree-elevated to degree $n_{1}+m_{1}$.

Proof. See [1].

The convergence of the approximate solution could be done in two ways.

1. Degree raising case of the Bezier polynomial approximation.

2. Subdivision case of the time interval.

In the following we prove the convergence in each case.

\subsection{Degree raising case}

Theorem 3.2. If time varying optimal control problem (8) has a unique, $C^{1}$ continuous trajectory solution $\bar{x}, C^{0}$ continuous control solution $\bar{u}$, then the approximate solution obtained by the control-point-based method converges to the exact solution as the degree of the approximate solution tends to infinity.

Proof. Given an arbitrary small positive number $\epsilon>0$, by the Weierstrass Theorem (see [19]) one can easily find polynomials $Q_{1, N_{1}}(t)$ of degree $N_{1}$ and $Q_{2, N_{2}}(t)$ of degree $N_{2}$ such that

$$
\left\|\frac{d^{i} Q_{1, N_{1}}(t)}{d t^{i}}-\frac{d^{i} \bar{x}(t)}{d t^{i}}\right\|_{\infty} \leq \frac{\epsilon}{16}, i=0,1, \quad \text { and }\left\|Q_{2, N_{2}}(t)-\bar{u}(t)\right\|_{\infty} \leq \frac{\epsilon}{16},
$$


where $\|\cdot\|_{\infty}$ stands for the $L_{\infty}$-norm over $[0,1]$. Especially, we have

$$
\begin{aligned}
\left\|a-Q_{1, N_{1}}(0)\right\|_{\infty} & \leq \frac{\epsilon}{16}, \\
\left\|b-Q_{1, N_{1}}(1)\right\|_{\infty} & \leq \frac{\epsilon}{16}, \\
\left\|a_{1}-Q_{2, N_{2}}(0)\right\|_{\infty} & \leq \frac{\epsilon}{16} .
\end{aligned}
$$

In general, $Q_{1, N_{1}}(t)$ and $Q_{2, N_{2}}(t)$ do not satisfy the boundary conditions. After a small perturbation with linear and constant polynomials $\alpha t+\beta$ and $\gamma$, respectively for $Q_{1, N_{1}}(t)$ and $Q_{2, N_{2}}(t)$, we can obtain polynomials $P_{1, N_{1}}(t)=$ $Q_{1, N_{1}}(t)+(\alpha t+\beta)$ and $P_{2, N_{2}}(t)=Q_{2, N_{2}}(t)+\gamma$ such that $P_{1, N_{1}}(t)$ satisfy the boundary conditions $P_{1, N_{1}}(0)=a, P_{1, N_{1}}(1)=b$, and $P_{2, N_{2}}(0)=a_{1}$. Thus $Q_{1, N_{1}}(0)+\beta=a$, and $Q_{1, N_{1}}(1)+\alpha+\beta=b$. By using (9), one have

$$
\begin{aligned}
\left\|a-Q_{1, N_{1}}(0)\right\|_{\infty} & =\|\beta\|_{\infty} \leq \frac{\epsilon}{16}, \\
\left\|b-Q_{1, N_{1}}(1)\right\|_{\infty} & =\|\alpha+\beta\|_{\infty} \leq \frac{\epsilon}{16} .
\end{aligned}
$$

Since

$$
\|\alpha\|_{\infty}-\|\beta\|_{\infty} \leq\|\alpha+\beta\|_{\infty} \leq \frac{\epsilon}{16},
$$

so

$$
\|\alpha\|_{\infty} \leq \frac{\epsilon}{16}+\|\beta\|_{\infty} \leq \frac{\epsilon}{16}+\frac{\epsilon}{16}=\frac{\epsilon}{8} .
$$

By the time, $a_{1}=P_{2, N_{2}}(0)=Q_{2, N_{2}}(0)+\gamma$, so

$$
\left\|a_{1}-Q_{2, N_{2}}(0)\right\|_{\infty}=\|\gamma\|_{\infty} \leq \frac{\epsilon}{16} .
$$

Now, we have

$$
\begin{aligned}
\left\|P_{1, N_{1}}(t)-\bar{x}(t)\right\|_{\infty}= & \left\|Q_{1, N_{1}}(t)+\alpha t+\beta-\bar{x}(t)\right\|_{\infty} \\
& \leq\left\|Q_{1, N_{1}}(t)-\bar{x}(t)\right\|_{\infty}+\|\alpha+\beta\|_{\infty} \leq \frac{\epsilon}{8}<\frac{\epsilon}{3}, \\
\left\|\frac{d P_{1, N_{1}}(t)}{d t}-\frac{d \bar{x}(t)}{d t}\right\|_{\infty}= & \left\|\frac{d Q_{1, N_{1}}(t)}{d t}+\alpha-\frac{d \bar{x}(t)}{d t}\right\|_{\infty} \\
\leq & \left\|\frac{d Q_{1, N_{1}}(t)}{d t}-\frac{d \bar{x}(t)}{d t}\right\|_{\infty}+\|\alpha\|_{\infty} \leq \frac{3 \epsilon}{16}<\frac{\epsilon}{3},
\end{aligned}
$$




$$
\begin{aligned}
\left\|P_{2, N_{2}}(t)-\bar{u}(t)\right\|_{\infty} & =\left\|Q_{2, N_{2}}(t)+\gamma-\bar{u}(t)\right\|_{\infty} \\
& \leq\left\|Q_{2, N_{2}}(t)-\bar{u}(t)\right\|_{\infty}+\|\gamma\|_{\infty} \leq \frac{\epsilon}{8}<\frac{\epsilon}{3} .
\end{aligned}
$$

Now, let define

$$
\begin{aligned}
L P_{N}(t) & =L\left(P_{1, N_{1}}(t), P_{2, N_{2}}(t), \dot{P}_{1, N_{1}}(t)\right) \\
& =\frac{d P_{1, N_{1}}(t)}{d t}-A(t) P_{1, N_{1}}(t)-B(t) P_{2, N_{2}}(t)=F(t),
\end{aligned}
$$

for every $t \in[0,1]$. Thus for $N \geq \max \left\{N_{1}, N_{2}\right\}$, one may find an upper bound for the following residual:

$$
\begin{aligned}
\left\|L P_{N}(t)-F(t)\right\|_{\infty}= & \left\|L\left(P_{1, N_{1}}(t), P_{2, N_{2}}(t), \dot{P}_{1, N_{1}}(t)\right)-F(t)\right\|_{\infty} \\
\leq & \left\|\frac{d P_{1, N_{1}}(t)}{d t}-\frac{d \bar{x}(t)}{d t}\right\|_{\infty}+\|A(t)\|_{\infty}\left\|P_{1, N_{1}}(t)-\bar{x}(t)\right\|_{\infty} \\
& +\|B(t)\|_{\infty}\left\|P_{2, N_{2}}(t)-\bar{u}(t)\right\|_{\infty} \\
\leq & C_{1}\left(\frac{\epsilon}{3}+\frac{\epsilon}{3}+\frac{\epsilon}{3}\right)=C_{1} \epsilon
\end{aligned}
$$

where $C_{1}=1+\|A(t)\|_{\infty}+\|B(t)\|_{\infty}$ is a constant.

Since the residual $R\left(P_{N}\right):=L P_{N}(t)-F(t)$ is a polynomial, we can represent it by a Bezier form. Thus we have

$$
R\left(P_{N}\right):=\sum_{i=0}^{m_{1}} d_{i, m_{1}} B_{i, m_{1}}(t) .
$$

Then from Lemma 1 in [1], there exists an integer $M(\geq N)$ such that when $m_{1}>M$, we have

$$
\left|\frac{1}{m_{1}+1} \sum_{i=0}^{m_{1}} d_{i, m_{1}}^{2}-\int_{0}^{1}\left(R\left(P_{N}\right)\right)^{2} d t\right|<\epsilon,
$$

which gives

$$
\frac{1}{m_{1}+1} \sum_{i=0}^{m_{1}} d_{i, m_{1}}^{2}<\epsilon+\int_{0}^{1}\left(R\left(P_{N}\right)\right)^{2} d t \leq \epsilon+C_{1}^{2} \epsilon^{2} .
$$


Suppose $x(t)$ and $u(t)$ are approximated solution of (8) obtained by the controlpoint-based method of degree $m_{2}\left(m_{2} \geq m_{1} \geq M\right)$. Let

$$
\begin{aligned}
R(x(t), u(t), \dot{x}(t)) & =L(x(t), u(t), \dot{x}(t))-F(t) \\
& =\sum_{i=0}^{m_{2}} c_{i, m_{2}} B_{i, m_{2}}(t), \quad m_{2} \geq m_{1} \geq M, \quad t \in[0,1] .
\end{aligned}
$$

Define the following norm for difference approximated solution $(x(t), u(t))$ and exact solution $(\bar{x}(t), \bar{u}(t))$ :

$$
\begin{aligned}
\|(x(t), u(t))-(\bar{x}(t), \bar{u}(t))\|:= & \int_{0}^{1} \sum_{j=0}^{1}\left|\frac{d^{j} x(t)}{d t^{j}}-\frac{d^{j} \bar{x}(t)}{d t^{j}}\right|^{2} d t \\
& +\int_{0}^{1}|u(0)-\bar{u}(0)| d t .
\end{aligned}
$$

It is easy to show that:

$$
\begin{aligned}
\|(x(t), u(t))-(\bar{x}(t), \bar{u}(t))\| \leq & C(|x(0)-\bar{x}(0)|+|x(1)-\bar{x}(1)| \\
& \left.+|u(0)-\bar{u}(0)|+\|R(x(t), u(t), \dot{x}(t))\|_{2}^{2}\right) \\
= & C \int_{0}^{1} \sum_{i=0}^{m_{2}}\left(c_{i, m_{2}} B_{i, m_{2}}(t)\right)^{2} d t \\
\leq & \frac{C}{m_{2}+1} \sum_{i=0}^{m_{2}} c_{i, m_{2}}^{2}
\end{aligned}
$$

Last inequality in (12) is obtained from Lemma 1 in [1] in which $C$ is a constant positive number. Now from Lemma 1 in [1], one can easily show that:

$$
\begin{aligned}
\|(x(t), u(t))-(\bar{x}(t), \bar{u}(t))\| & \leq \frac{C}{m_{2}+1} \sum_{i=0}^{m_{2}} c_{i, m_{2}}^{2} \leq \frac{C}{m_{2}+1} \sum_{i=0}^{m_{2}} d_{i, m_{2}}^{2} \\
& \leq \frac{C}{m_{1}+1} \sum_{i=0}^{m_{1}} d_{i, m_{1}}^{2} \leq C\left(\epsilon+C_{1}^{2} \epsilon^{2}\right) \\
& =\epsilon_{1}, m_{1} \geq M,
\end{aligned}
$$

where last inequality in (13) is coming from (10). 
Thus, from (13) we have:

$$
\begin{aligned}
\|x(t)-\bar{x}(t)\| & \leq \epsilon_{1}, \\
\|u(t)-\bar{u}(t)\| & \leq \epsilon_{1} .
\end{aligned}
$$

Since the infinite norm and the norm defined in (11) are equivalent, there is a $\rho_{1}>0$ where

$$
\begin{aligned}
\|x(t)-\bar{x}(t)\|_{\infty} & \leq \rho_{1} \epsilon_{1}, \\
\|u(t)-\bar{u}(t)\|_{\infty} & \leq \rho_{1} \epsilon_{1} .
\end{aligned}
$$

Now, we show that the approximated cost function tends to exact cost function as the degree of Bezier approximation increases. Define

$$
\begin{aligned}
I_{\text {exact }}= & \frac{1}{2} \bar{x}(1) H(1) \bar{x}(1)+\int_{0}^{1}(\bar{x}(t) P(t) \bar{x}(t)+\bar{u}(t) Q(t) \bar{u}(t) \\
& +K(t) \bar{x}(t)+R(t) \bar{u}(t)) d t, \\
I_{\text {approx }}= & \frac{1}{2} x(1) H(1) x(1)+\int_{0}^{1}(x(t) P(t) x(t)+u(t) Q(t) u(t) \\
& +K(t) x(t)+R(t) u(t)) d t,
\end{aligned}
$$

for $t \in[0,1]$. Now, there are four positive integers $M_{i} \geq 0, i=1, \ldots, 6$, such that

$$
\begin{gathered}
\|P(t)\|_{\infty} \leq M_{1}, \quad\|Q(t)\|_{\infty} \leq M_{2}, \quad\|K(t)\|_{\infty} \leq M_{3}, \\
\|R(t)\|_{\infty} \leq M_{4}, \quad\|\bar{x}(t)\|_{\infty} \leq M_{5} \quad \text { and } \quad\|\bar{u}(t)\|_{\infty} \leq M_{6} .
\end{gathered}
$$

Since

$$
\begin{aligned}
\|x(t)\|_{\infty}-\|\bar{x}(t)\|_{\infty} & \leq\|\bar{x}(t)-x(t)\|_{\infty} \leq \rho_{1} \epsilon_{1}, \\
\|u(t)\|_{\infty}-\|\bar{u}(t)\|_{\infty} & \leq\|\bar{u}(t)-u(t)\|_{\infty} \leq \rho_{1} \epsilon_{1},
\end{aligned}
$$

we have

$$
\begin{aligned}
\|x(t)\|_{\infty} & \leq\|\bar{x}(t)\|_{\infty}+\rho_{1} \epsilon_{1} \leq M_{5}+\rho_{1} \epsilon_{1}, \\
\|u(t)\|_{\infty} & \leq\|\bar{u}(t)\|_{\infty}+\rho_{1} \epsilon_{1} \leq M_{6}+\rho_{1} \epsilon_{1},
\end{aligned}
$$


so

$$
\begin{aligned}
\|\bar{x}(t)+x(t)\|_{\infty} & \leq\|\bar{x}(t)\|_{\infty}+\|x(t)\|_{\infty} \leq 2 M_{5}+\rho_{1} \epsilon_{1}, \\
\|\bar{u}(t)+u(t)\|_{\infty} & \leq\|\bar{u}(t)\|_{\infty}+\|u(t)\|_{\infty} \leq 2 M_{6}+\rho_{1} \epsilon_{1},
\end{aligned}
$$

now, we have

$$
\begin{aligned}
& \left\|I_{\text {exact }}-I_{\text {approx }}\right\|_{\infty}=\| \int_{0}^{1} \bar{x}(t) P(t) \bar{x}(t)+\bar{u}(t) Q(t) \bar{u}(t)+K(t) \bar{x}(t) \\
& \quad+R(t) \bar{u}(t)-x(t) P(t) x(t)-u(t) Q(t) u(t)-K(t) x(t)-R(t) u(t) d t \|_{\infty} \\
& \leq \int_{0}^{1}\|\bar{x}(t) P(t) \bar{x}(t)-x(t) P(t) x(t)\|_{\infty} d t \\
& \quad+\int_{0}^{1}\|\bar{u}(t) Q(t) \bar{u}(t)-u(t) Q(t) u(t)\|_{\infty} d t \\
& \quad+\int_{0}^{1}\|K(t) \bar{x}(t)-K(t) x(t)\|_{\infty} d t+\int_{0}^{1}\|R(t) \bar{u}(t)-R(t) u(t)\|_{\infty} d t \\
& \leq \int_{0}^{1}\|P(t)\|_{\infty}\left\|\bar{x}^{2}(t)-x^{2}(t)\right\|_{\infty} d t+\int_{0}^{1}\|Q(t)\|_{\infty}\left\|\bar{u}^{2}(t)-u^{2}(t)\right\|_{\infty} d t \\
& \leq \int_{0}^{1}\|P(t)\|_{\infty}\|\bar{x}(t)-x(t)\|_{\infty}\left\|_{\bar{x}}(t)+x(t)\right\|_{\infty} d t \\
& \quad+\int_{0}^{1}\|Q(t)\|_{\infty}\|\bar{u}(t)-u(t)\|_{\infty}\|\bar{u}(t)+u(t)\|_{\infty} d t \\
& \quad+\int_{0}^{1}\|K(t)\|_{\infty}\|\bar{x}(t)-x(t)\|_{\infty} d t+\int_{0}^{1}\|R(t)\|_{\infty}\|\bar{u}(t)-u(t)\|_{\infty} d t \\
& \leq M_{1} \rho_{1} \epsilon_{1}\left(\rho_{1} \epsilon_{1}+2 M_{5}\right)+M_{2} \rho_{1} \epsilon_{1}\left(\rho_{1} \epsilon_{1}+2 M_{6}\right)+M_{3} \rho_{1} \epsilon_{1}+M_{4} \rho_{1} \epsilon_{1} .
\end{aligned}
$$

This completes the proof.

\subsection{Subdivision case}

Theorem 3.3. Let $(x, u)$ be the approximate solution of the linear optimal control problem (8) obtained by the subdivision scheme of the control-pointbased method. If (8) has a unique solution $(\bar{x}, \bar{u})$ where $(\bar{x}, \bar{u})$ is smooth enough so that the cubic spline $T(\bar{x}, \bar{u})$ interpolating to $(\bar{x}, \bar{u})$ converges to $(\bar{x}, \bar{u})$ in the order $O\left(\tau^{q}\right),(q>2)$, where $\tau$ is the maximal width of all subintervals, then $(x, u)$ converges to $(\bar{x}, \bar{u})$ as $\tau \rightarrow 0$. 
Proof. We first impose a uniform partition $\prod_{d}=\bigcup_{i}\left[t_{i}, t_{i+1}\right]$ on the interval $[0,1]$ as $t_{i}=i d$ where $d=\frac{1}{n_{1}+1}$.

Let $I_{d}\left(\bar{x}(t), \bar{u}(t), \frac{d \bar{x}(t)}{d t}\right)$ be the cubic spline over $\prod_{d}$ interpolating to $(\bar{x}, \bar{u})$. Then for an arbitrary small positive number $\epsilon>0$, there exists a $\delta_{1}>0$ such that

$$
\left\|L\left(\bar{x}(t), \bar{u}(t), \frac{d \bar{x}(t)}{d t}\right)-L\left(I_{d}\left(\bar{x}(t), \bar{u}(t), \frac{d \bar{x}(t)}{d t}\right)\right)\right\|_{\infty} \leq \epsilon
$$

provided that $d<\delta_{1}$. Let

$$
R\left(I_{d}\left(\bar{x}(t), \bar{u}(t), \frac{d \bar{x}(t)}{d t}\right)\right)=L\left(I_{d}\left(\bar{x}(t), \bar{u}(t), \frac{d \bar{x}(t)}{d t}\right)\right)-F(t)
$$

be the residual. For each subinterval $\left[t_{i}, t_{i+1}\right], R\left(I_{d}\left(\bar{x}(t), \bar{u}(t), \frac{d \bar{x}(t)}{d t}\right)\right)$ is a polynomial. On each interval $\left[t_{i}, t_{i+1}\right]$, we impose another uniform partition $\prod_{i, \tau}=\bigcup_{j}\left[t_{i, j}, t_{i, j+1}\right]$ as $t_{i, j}=i d+j \tau$ where $\tau=\frac{d}{m_{1}}, j=0, \ldots, m_{1}$. Express $R\left(I_{d}\left(\bar{x}(t), \bar{u}(t), \frac{d \bar{x}(t)}{d t}\right)\right)$ in $\left[t_{i, j-1}, t_{i, j}\right]$ as

$$
R\left(I_{d}\left(\bar{x}(t), \bar{u}(t), \frac{d \bar{x}(t)}{d t}\right)\right)=\sum_{p_{1}=0}^{l} r_{p_{1}}^{i, j} B_{p_{1}, l}(t), \quad t \in\left[t_{i, j-1}, t_{i, j}\right] .
$$

By Lemma 3 in [1], there exists a $\delta_{2}>0\left(\delta_{2} \leq \delta_{1}\right)$ such that when $\tau<\delta_{2}$, we have

$$
\left|\sum_{j=1}^{m_{1}}\left(t_{i, j}-t_{i, j-1}\right) \sum_{p_{1}=0}^{l}\left(r_{p_{1}}^{i, j}\right)^{2}(l+1) \int_{t_{i}}^{t_{i+1}} R^{2}\left(I_{d}\left(\bar{x}(t), \bar{u}(t), \frac{d \bar{x}(t)}{d t}\right)\right)\right| \leq \frac{\epsilon}{d} .
$$

Thus

$$
\left|\sum_{i=1}^{n_{1}} \sum_{j=1}^{m_{1}}\left(t_{i, j}-t_{i, j-1}\right) \sum_{p_{1}=0}^{l}\left(r_{p_{1}}^{i, j}\right)^{2}(l+1) \int_{0}^{1} R^{2}\left(I_{d}\left(\bar{x}(t), \bar{u}(t), \frac{d \bar{x}(t)}{d t}\right)\right)\right| \leq \epsilon,
$$

or

$$
\begin{aligned}
\sum_{i=1}^{n_{1}} \sum_{j=1}^{m_{1}}\left(t_{i, j}-t_{i, j-1}\right) \sum_{p_{1}=0}^{l}\left(r_{p_{1}}^{i, j}\right)^{2} & <(l+1) \int_{0}^{1} R^{2}\left(I_{d}\left(\bar{x}(t), \bar{u}(t), \frac{d \bar{x}(t)}{d t}\right)+\epsilon\right. \\
& <(l+1) \epsilon^{2}+\epsilon .
\end{aligned}
$$


Now combining the partitions $\prod_{d}$ and all $\prod_{i, \tau}$ gives a denser partition with the length $\tau$ for each subinterval. Suppose $(x(t), u(t))$ is the approximate solution by the control-point-based method with respect to this partition, and denote the residual over $\left[t_{i, j-1}, t_{i, j}\right]$ by

$$
R\left(x(t), u(t), \frac{d x(t)}{d t}\right)=L\left(x(t), u(t), \frac{d x(t)}{d t}\right)-F(t)=\sum_{p_{1}=0}^{l} c_{p_{1}}^{i, j} B_{p_{1}, l}(t) .
$$

Then there is a constant $C$ such that

$$
\begin{aligned}
& \|(x(t), u(t))-(\bar{x}(t), \bar{u}(t))\| \\
& \leq C\left\|R\left(\left(x(t), u(t), \frac{d x(t)}{d t}\right)-\left(\bar{x}(t), \bar{u}(t), \frac{d \bar{x}(t)}{d t}\right)\right)\right\|_{2}^{2} \\
& \quad \leq \frac{C}{l+1} \sum_{i=1}^{n} \sum_{j=1}^{m}\left(t_{i, j}-t_{i, j-1}\right) \sum_{p_{1}=0}^{l}\left(c_{p_{1}}^{i, j}\right)^{2}
\end{aligned}
$$

last inequality in (14) is obtained from Lemma 1 in [1]. One can easily show that:

$$
\begin{aligned}
\|(x(t), u(t))-(\bar{x}(t), \bar{u}(t))\| & \leq \frac{C}{l+1} \sum_{i=1}^{n_{1}} \sum_{j=1}^{m_{1}}\left(t_{i, j}-t_{i, j-1}\right) \sum_{p_{1}=0}^{l}\left(c_{p_{1}}^{i, j}\right)^{2} \\
& \leq \frac{C}{l+1} \sum_{i=1}^{n_{1}} \sum_{j=1}^{m_{1}}\left(t_{i, j}-t_{i, j-1}\right) \sum_{p_{1}=0}^{l}\left(r_{p_{1}}^{i, j}\right)^{2} \\
& \leq C\left(\epsilon^{2}+\frac{\epsilon}{l+1}\right)=\epsilon_{2} .
\end{aligned}
$$

Thus, from (15) we have:

$$
\begin{aligned}
&\|x(t)-\bar{x}(t)\| \leq \epsilon_{2}, \\
&\|u(t)-\bar{u}(t)\| \leq \epsilon_{2} .
\end{aligned}
$$

Since the infinite norm and the norm defined in (11) are equivalent, there is a $\rho_{2}>0$ where

$$
\begin{aligned}
\|x(t)-\bar{x}(t)\|_{\infty} & \leq \rho_{2} \epsilon_{2}, \\
\|u(t)-\bar{u}(t)\|_{\infty} & \leq \rho_{2} \epsilon_{2} .
\end{aligned}
$$


Now, we show that the approximated cost function tends to exact cost function as the degree of approximation increases. Define

$$
\begin{aligned}
I_{\text {exact }}= & \frac{1}{2} \bar{x}(1) H(1) \bar{x}(1)+\int_{0}^{1}(\bar{x}(t) P(t) \bar{x}(t)+\bar{u}(t) Q(t) \bar{u}(t) \\
& +K(t) \bar{x}(t)+R(t) \bar{u}(t)) d t, \\
I_{\text {approx }}= & \frac{1}{2} x(1) H(1) x(1)+\int_{0}^{1}(x(t) P(t) x(t)+u(t) Q(t) u(t) \\
& +K(t) x(t)+R(t) u(t)) d t,
\end{aligned}
$$

for $t \in\left[t_{i, j-1}, t_{i, j}\right]$. Now, there are four positive integers $M_{i} \geq 0, i=1, \ldots, 6$, such that $\|P(t)\|_{\infty} \leq M_{1},\|Q(t)\|_{\infty} \leq M_{2},\|K(t)\|_{\infty} \leq M_{3},\|R(t)\|_{\infty} \leq M_{4}$, $\|\bar{x}(t)\|_{\infty} \leq M_{5}$, and $\|\bar{u}(t)\|_{\infty} \leq M_{6}$. Since

$$
\begin{aligned}
\|x(t)\|_{\infty}-\|\bar{x}(t)\|_{\infty} & \leq\|\bar{x}(t)-x(t)\|_{\infty} \leq \rho_{2} \epsilon_{2}, \\
\|u(t)\|_{\infty}-\|\bar{u}(t)\|_{\infty} & \leq\|\bar{u}(t)-u(t)\|_{\infty} \leq \rho_{2} \epsilon_{2},
\end{aligned}
$$

we have

$$
\begin{aligned}
\|x(t)\|_{\infty} & \leq\|\bar{x}(t)\|_{\infty}+\rho_{2} \epsilon_{2} \leq M_{5}+\rho_{2} \epsilon_{2}, \\
\|u(t)\|_{\infty} & \leq\|\bar{u}(t)\|_{\infty}+\rho_{2} \epsilon_{2} \leq M_{6}+\rho_{2} \epsilon_{2},
\end{aligned}
$$

so

$$
\begin{aligned}
\|\bar{x}(t)+x(t)\|_{\infty} & \leq\|\bar{x}(t)\|_{\infty}+\|x(t)\|_{\infty} \leq 2 M_{5}+\rho_{2} \epsilon_{2}, \\
\|\bar{u}(t)+u(t)\|_{\infty} & \leq\|\bar{u}(t)\|_{\infty}+\|u(t)\|_{\infty} \leq 2 M_{6}+\rho_{2} \epsilon_{2},
\end{aligned}
$$

now, we have

$$
\begin{aligned}
& \left\|I_{\text {exact }}-I_{\text {approx }}\right\|_{\infty}=\| \sum_{i=1}^{n_{1}} \sum_{j=1}^{m_{1}} \int_{t_{i, j-1}}^{t_{i, j}} \bar{x}(t) P(t) \bar{x}(t)+\bar{u}(t) Q(t) \bar{u}(t)+K(t) \bar{x}(t) \\
& \quad+R(t) \bar{u}(t)-x(t) P(t) x(t)-u(t) Q(t) u(t)-K(t) x(t)-R(t) u(t) d t \|_{\infty} \\
& \leq \sum_{i=1}^{n_{1}} \sum_{j=1}^{m_{1}} \int_{t_{i, j-1}}^{t_{i, j}}\|\bar{x}(t) P(t) \bar{x}(t)-x(t) P(t) x(t)\|_{\infty} d t
\end{aligned}
$$




$$
\begin{aligned}
& +\sum_{i=1}^{n_{1}} \sum_{j=1}^{m_{1}} \int_{t_{i, j-1}}^{t_{i, j}}\|\bar{u}(t) Q(t) \bar{u}(t)-u(t) Q(t) u(t)\|_{\infty} d t \\
& +\sum_{i=1}^{n_{1}} \sum_{j=1}^{m_{1}} \int_{t_{i, j-1}}^{t_{i, j}}\|K(t) \bar{x}(t)-K(t) x(t)\|_{\infty} d t \\
& +\sum_{i=1}^{n} \sum_{j=1}^{m} \int_{t_{i, j-1}}^{t_{i, j}}\|R(t) \bar{u}(t)-R(t) u(t)\|_{\infty} d t \\
& \leq \sum_{i=1}^{n_{1}} \sum_{j=1}^{m_{1}} \int_{t_{i, j-1}}^{t_{i, j}}\|P(t)\|_{\infty}\left\|\bar{x}^{2}(t)-x^{2}(t)\right\|_{\infty} d t \\
& +\sum_{i=1}^{n_{1}} \sum_{j=1}^{m_{1}} \int_{t_{i, j-1}}^{t_{i, j}}\|Q(t)\|_{\infty}\left\|\bar{u}^{2}(t)-u^{2}(t)\right\|_{\infty} d t \\
& \leq \sum_{i=1}^{n_{1}} \sum_{j=1}^{m_{1}} \int_{t_{i, j-1}}^{t_{i, j}}\|P(t)\|_{\infty}\|\bar{x}(t)-x(t)\|_{\infty}\|\bar{x}(t)+x(t)\|_{\infty} d t \\
& +\sum_{i=1}^{n_{1}} \sum_{j=1}^{m_{1}} \int_{t_{i, j-1}}^{t_{i, j}}\|Q(t)\|_{\infty}\|\bar{u}(t)-u(t)\|_{\infty}\|\bar{u}(t)+u(t)\|_{\infty} d t \\
& +\sum_{i=1}^{n_{1}} \sum_{j=1}^{m_{1}} \int_{t_{i, j-1}}^{t_{i, j}}\|K(t)\|_{\infty}\|\bar{x}(t)-x(t)\|_{\infty} d t \\
& +\sum_{i=1}^{n_{1}} \sum_{j=1}^{m_{1}} \int_{t_{i, j-1}}^{t_{i, j}}\|R(t)\|_{\infty}\|\bar{u}(t)-u(t)\|_{\infty} d t \\
& \leq \frac{n_{1}\left(n_{1}+1\right)}{2} \frac{m_{1}\left(m_{1}+1\right)}{2} \tau M_{1} \rho_{2} \epsilon_{2}\left(\rho_{2} \epsilon_{2}+2 M_{5}\right) \\
& +\frac{n_{1}\left(n_{1}+1\right)}{2} \frac{m_{1}\left(m_{1}+1\right)}{2} \tau M_{2} \rho_{2} \epsilon_{2}\left(\rho_{2} \epsilon_{2}+2 M_{6}\right)+M_{3} \rho_{2} \epsilon_{2}+M_{4} \rho_{2} \epsilon_{2} .
\end{aligned}
$$

Now, from Lemma 3 in [1], we conclude that the approximated solution converges to the exact solution in order $o\left(\tau^{q}\right),(q>2)$.

This completes the proof. 


\section{Numerical examples}

Example 4.1. Consider the following constrained optimal control problem (see [5]):

$$
\begin{aligned}
\min I= & \int_{0}^{3} 2 x_{1}(t) d t \\
\text { s.t. } & \dot{x}_{1}(t)=x_{2}(t) \\
& \dot{x}_{2}(t)=u(t) \\
& -2 \leq u(t) \leq 2 \\
& x_{1}(t) \geq-6 \\
& x_{1}(0)=2, x_{2}(0)=0
\end{aligned}
$$

Let $k=6$ and, $n=3$, if one consider the $C^{1}$ continuity of $u(t)$, one can find the following solution from (7)

$$
u(t)= \begin{cases}-1.997033122-0.05933753750 t+0.3204226750 t^{2}-0.4272302200 t^{3} & 0 \leq t \leq 0.5, \\ -1.895851300-0.4017993675 t+0.4760881250 t^{2}-0.1781683800 t^{3} & 0.5 \leq t \leq 1, \\ -1.815689262-0.5715973000 t+0.5751978700 t^{2}-0.1876422300 t^{3} & 1 \leq t \leq 1.5, \\ -5.617179042+10.40916179 t-8.997161210 t^{2}+2.439960460 t^{3} & 1.5 \leq t \leq 2, \\ 19.95368871-36.19728204 t+18.43113180 t^{2}-2.818933558 t^{3} & 2 \leq t \leq 2.5, \\ 103.0503639-156.4637034 t+74.75786477 t^{2}-11.42518654 t^{3} & 2.5 \leq t \leq 3 .\end{cases}
$$

Then by involving the above control function $u($.$) , in the indicated differential$ equation, one can find the trajectories $x_{1}($.$) and x_{2}($.$) as follows:$

$$
x_{1}(t)=\left\{\begin{array}{lc}
-.998516561 t^{2}-0.0098895895 t^{3}+.0267018895 t^{4}-.021361511 t^{5}+2 & 0 \leq t \leq 0.5 \\
-.947925649 t^{2}-.0669665625 t^{3}+.0396740105 t^{4}-.008908419 t^{5}-.02251014 t+2.001950237 & 0.5 \leq t \leq 1 \\
-.90784463 t^{2}-.09526621483 t^{3}+.04793315525 t^{4}-.0093821114 t^{5}-.048441339 t+2.008314617 & 1 \leq t \leq 1.5 \\
-2.808589511 t^{2}+1.734860295 t^{3}-.749763434 t^{4}+.1219980229 t^{5}+.741484077 t+1.961919191 & 1.5 \leq t \leq 2 \\
9.976844345 t^{2}-6.032880343 t^{3}+1.53592765 t^{4}-.1409466778 t^{5}-9.293902341 t+4.874893216 & 2 \leq t \leq 2.5 \\
51.525182 t^{2}-26.07728389 t^{3}+6.229822062 t^{4}-.5712593272 t^{5}-50.5251822 t+20.1372572 & 2.5 \leq t \leq 3
\end{array}\right.
$$

and,

$$
x_{2}(t)= \begin{cases}-1.997033122 t-0.02966876850 t^{2}+.1068075580 t^{3}-.1068075550 t^{4} & 0 \leq t \leq 0.5 \\ -1.895851298 t-0.2008996875 t^{2}+.1586960420 t^{3}-0.04454209500 t^{4}-0.022510140 & 0.5 \leq t \leq 1 \\ -1.815689260 t-0.2857986445 t^{2}+.1917326210 t^{3}-0.04691055700 t^{4}-0.048441339 & 1 \leq t \leq 1.5 \\ -5.617179022 t+5.204580886 t^{2}-2.999053736 t^{3}+.6099901145 t^{4}+.741484077 & 1.5 \leq t \leq 2, \\ 19.95368869 t-18.09864103 t^{2}+6.143710602 t^{3}-.7047333890 t^{4}-9.293902341 & 2 \leq t \leq 2.5 \\ 103.0503640 t-78.23185168 t^{2}+24.91928825 t^{3}-2.856296636 t^{4}-50.52518220 & 2.5 \leq t \leq 3\end{cases}
$$


The graphs of approximated trajectories $x_{1}(t)$ and $x_{2}(t)$ are shown respectively in Figure 1 and Figure 2, and the graph of approximated control is shown Figure 3. The approximated and exact objective function are respectively $I=$ $-5.389857789, I^{*}=-5.528595476$ (see [5]).

In this example, we used Bezier polynomials of degree 10 to approximate the trajectories $x_{1}($.$) and x_{2}($.$) through the time interval J=[0,3]$, and without using subintervals. The objective function is found $I=-5.360252730$ and the trajectories $x_{1}(t)$, and $x_{2}(t)$ for $t \in J$ are shown in Figures 4, 5. For reduction in complicated manipulations, the authors recommend to use low degree Bezier approximation polynomials and use subintervals approach.

Example 4.2. Consider the following optimal control problem ([17]):

$$
\begin{aligned}
\min I= & \int_{0}^{1}\left(x_{1}^{2}(t)+x_{2}^{2}(t)+0.005 u^{2}(t)\right) d t \\
\text { s.t. } & \dot{x}_{1}(t)=x_{2}(t) \\
\dot{x}_{2}(t) & =-x_{2}(t)+u(t) \\
x_{2}(t) & \leq q(t)=8(t-0.5)^{2}-0.5 \\
x_{1}(0) & =0, x_{2}(0)=-1
\end{aligned}
$$

Let $k=12, t_{0}=0, t_{f}=1, t_{j}=t_{0}+\frac{\left(t_{f}-t_{0}\right) j}{12}=\frac{j}{12},(j=1, \ldots, 12)$, and $n=3$. From (7), one can find the following solution

$$
u(t)= \begin{cases}11.43511691-76.63782944 t-44.52584083 t^{2}+0.0035860000 t^{3} & 0 \leq t \leq \frac{1}{12}, \\ 7.861400876-35.62214319 t-22.10043759 t^{2}+0.021243000 t^{3} & \frac{1}{12} \leq t \leq \frac{1}{6}, \\ 8.180459190-37.33660935 t-23.29089144 t^{2}-0.031848700 t^{3} & \frac{1}{6} \leq t \leq \frac{1}{4}, \\ -1.652748711-3.681790087 t-.5992407498 t^{2}+0.049746000 t^{3} & \frac{1}{4} \leq t \leq \frac{1}{3}, \\ -6.522781102+8.110829441 t+7.889653090 t^{2}-0.059637000 t^{3} & \frac{1}{3} \leq t \leq \frac{5}{12}, \\ -6.503100780+8.011432661 t+7.992145038 t^{2}-0.00515290 t^{3} & \frac{5}{12} \leq t \leq \frac{1}{2}, \\ -6.497644189+7.992176154 t+8.004580588 t^{2}+0.003349300 t^{3} & \frac{1}{2} \leq t \leq \frac{7}{12}, \\ -6.493920361+7.982943896 t+8.007520923 t^{2}+0.00668000 t^{3} & \frac{7}{12} \leq t \leq \frac{2}{3}, \\ 3.602879581+0.4707623628 t-3.420687999 t^{2}-0.02529800 t^{3} & \frac{2}{3} \leq t \leq \frac{3}{4}, \\ 11.39254692-3.778190612 t-11.63683471 t^{2}+0.0188618000 t^{3} & \frac{3}{4} \leq t \leq \frac{5}{6}, \\ .9495509436-0.1355315210 t-.9484970841 t^{2}-0.0070753900 t^{3} & \frac{5}{6} \leq t \leq \frac{11}{12}, \\ 0.4749655029-0.08216181395 t-0.4509062307 t^{2}+0.0027248400 t^{3} & \frac{11}{12} \leq t \leq 1,\end{cases}
$$




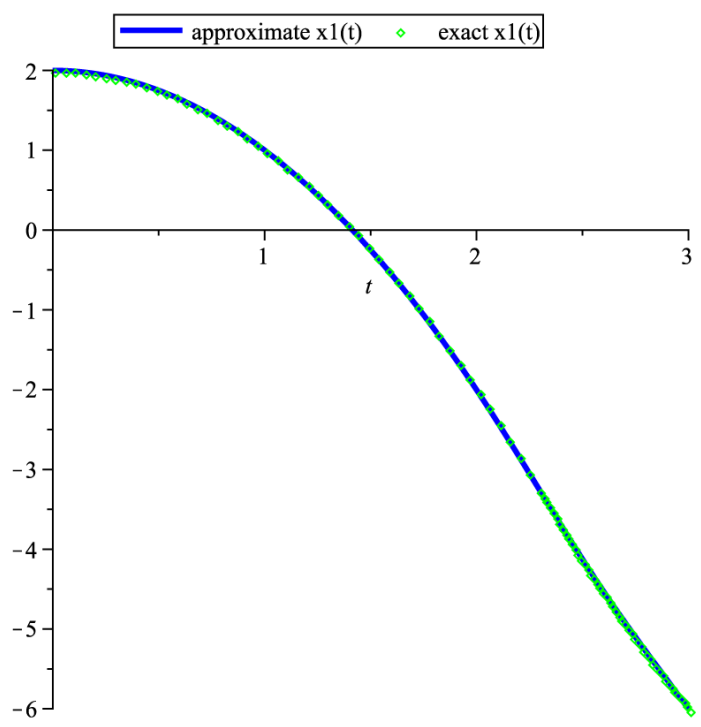

Figure 1 - The graphs of approximated and exact trajectories $x_{1}(t)$ for Example 4.1.

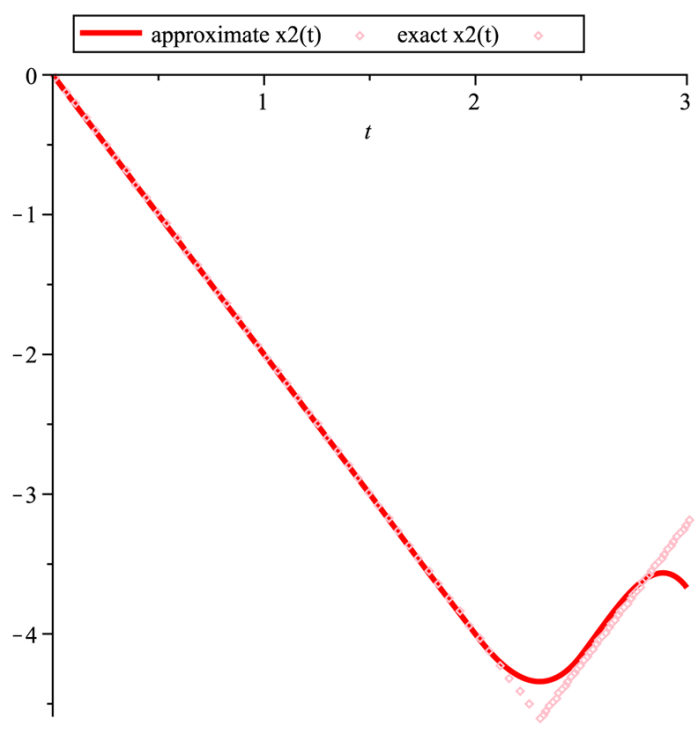

Figure 2 - The graphs of approximated and exact trajectories $x_{2}(t)$ for Example 4.1. 


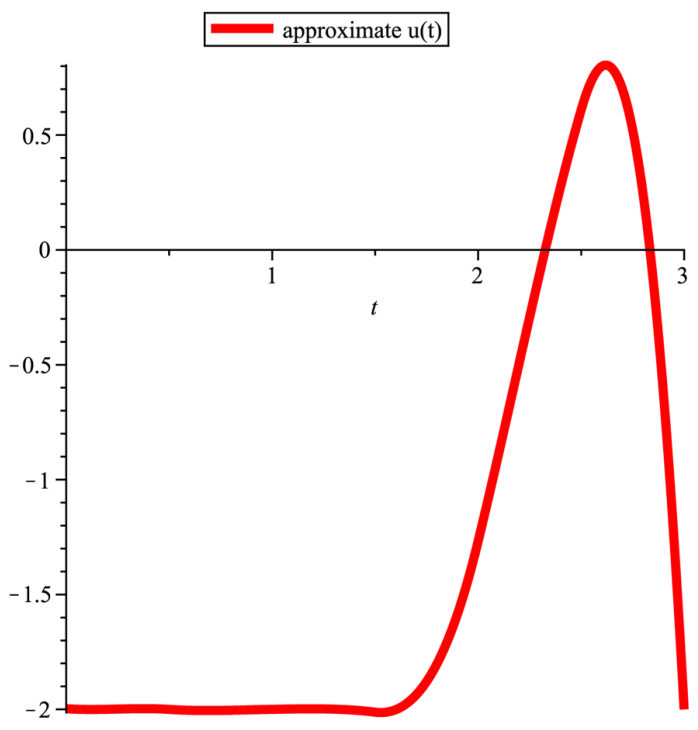

Figure 3 - The graph of approximated control for Example 4.1.

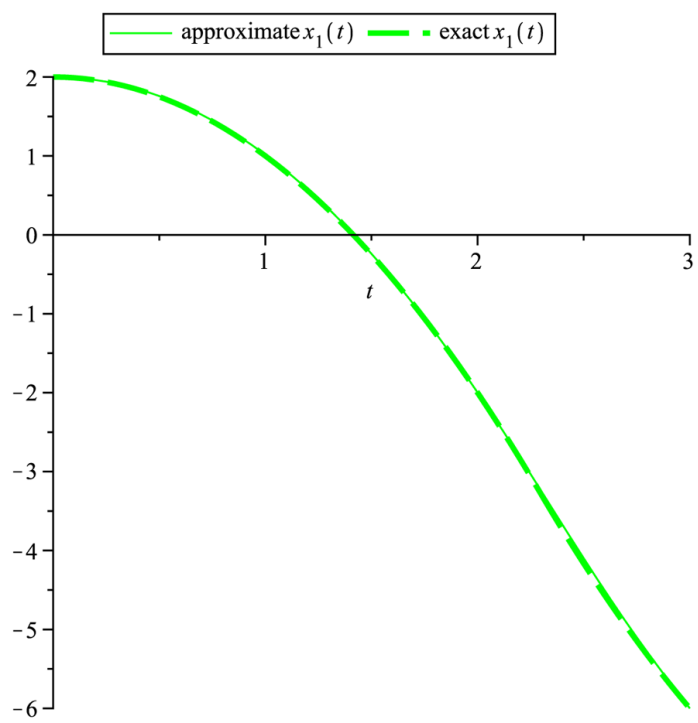

Figure 4 - The graphs of approximated and exact trajectories $x_{1}(t)$ for Example 4.1. 


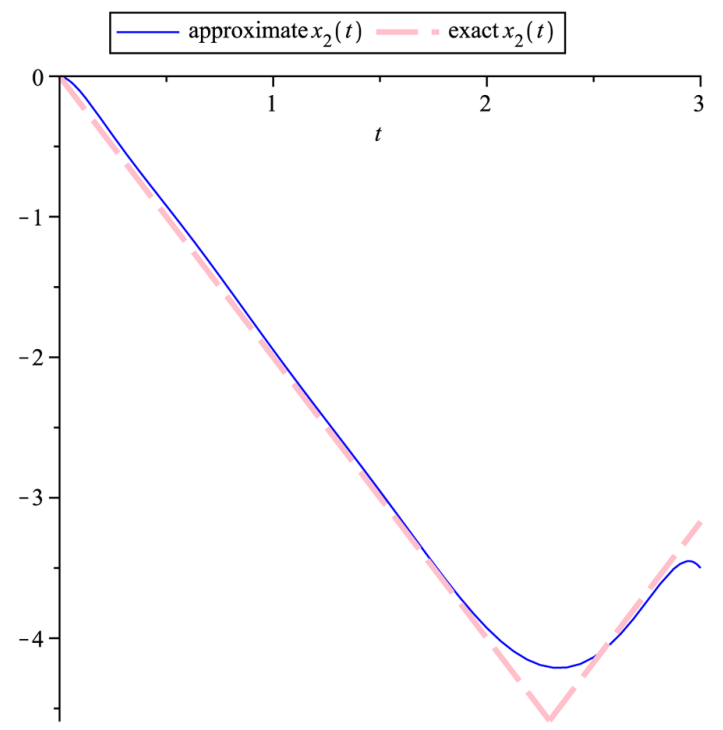

Figure 5 - The graphs of approximated and exact trajectories $x_{2}(t)$ for Example 4.1.

The graphs of approximated trajectories $x_{1}(t)$ and $x_{2}(t)$ are shown respectively in Figure 6 and Figure 7, and the graph of approximated control is shown Figure 8 . The approximated and exact objective function respectively are $I=$ $0.1728904530, I^{*}=0.17078488$ (see [17]). The computation takes 5 seconds of CPU time when it is performed using Maple 12 on an AMD Athelon X4 PC with 2 GB of RAM. The QPSolve command solves (7), which involves computing the minimum of a quadratic objective function possibly subject to linear constraints. The QPSolve command uses an iterative active- set method implemented in a built-in library provided by the numerical algorithms group.

\section{Conclusions}

In this paper, a Bézier control points method for solving optimal control problems governed by time varying linear dynamical systems with terminal state constraints and inequality constraints on the states and control has been suggested. The method replaces the constrained optimal control problem by a quadratic programming one, and the control point structure provides a bound on the residual function. The new mathematical programming problem is intuitive and easy to solve. 


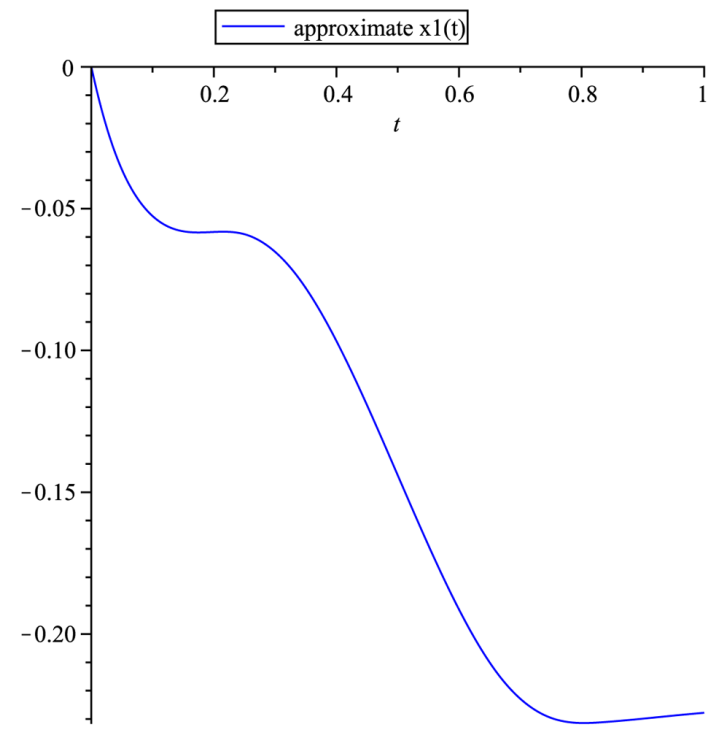

Figure 6 - The graph of approximated trajectory $x_{1}(t)$ for Example 4.2.

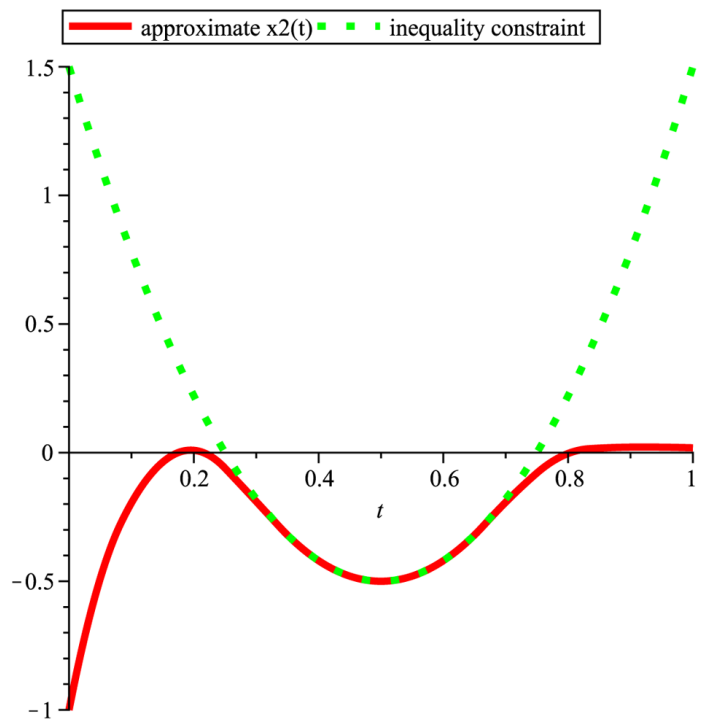

Figure 7 - The graphs of approximated trajectory $x_{2}(t)$ and $q(t)=8(t-0.5)^{2}-0.5$ for Example 4.2. 


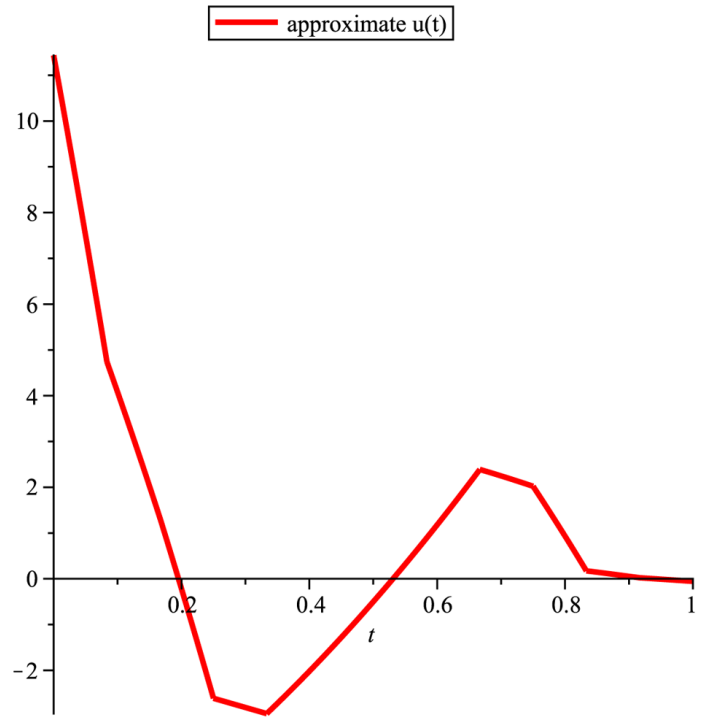

Figure 8 - The graph of approximated control for Example 4.2.

Numerical examples show that the proposed method is reliable and efficient. The control polygon gives an intermediate approximation to the appearance of the solution.

Acknowledgment. The authors like to express their sincere gratitude to referees for their very contractive advises.

\section{REFERENCES}

[1] J. Zheng, T.W. Sederberg and R.W. Johnson, Least squares methods for solving differential equations using Bezier control points. Appl. Num. Math, 48 (2004), 237-252.

[2] M. Razaghi and G. Elnagar, A legendre technique for solving time-varying linear quadratic optimal control problems. J. Franklin Institute, 330 (1993), 453-463.

[3] G.N. Elnagar and M. Razzaghi, A Collocation-type method for linear quadratic optimal control problems. Optimal. Control. Appl. Methods, 18 (1997), 227-235.

[4] L.A. Rodriguez and A. Sideris, A sequential linear quadratic approach for constrained nonlinear optimal control. American Control Conference, San Francisco, CA, USA (2011). 
[5] J. Vlassenbroeck, A Chebyshev polynomial method for optimal control with state constraints. Automatica, 24 (1988), 499-504.

[6] M. Razaghi and S. Yousefi, Legendre wavelets method for constrained optimal control problems. Mathematics Methods in the Applied Sciences, 25 (2002), 529539.

[7] M. Gachpazan, Solving of time varying quadratic optimal control problems by using Bézier control points. Computational \& Applied Mathematics, 30(2) (2011), 367-379.

[8] V. Yen and M. Nagurka, Optimal control of linearly constrained linear systems via state parameterization. Optimal Control. Appl. Methods, 13 (1992), 155-167.

[9] G. Elnagar, M. Kazemi and M. Razzaghi, The pseudospectral Legendre method for discretizing optimal control problem. IEEE Trans. Automat. Control., 40 (1965), 1793-1796.

[10] P.A. Frick and D.J. Stech, Solution of optimal control problems on a parallel machine using the Epsilon method. Optimal Control. Appl, 16 (1995), 1-17.

[11] C.P. Neuman and A. Sen, A suboptimal control algorithm for constrained problems using cubic splines. Automatica, 9 (1973), 601-613.

[12] R. Pytlak, Numerical methods for optimal control problems with state constraints. First ed, Berlin: Springer-Veriag (1999).

[13] H.R. Sirisena, Computation of optimal controls using a piecewise polynomial parameterization. IEEE Trans. Automat. Control, 18 (1973), 409-411.

[14] H.R. Sirisena and F.S. Chou, State parameterization approach to the solution of optimal control problems. Optimal Control. Appl. Methods, 2 (1981), 289-298.

[15] K. Teo, C. Goh and K. Wong, A unified computational approach to optimal control problems. First edition, London: Longman, Harlow (1981).

[16] I. Troch, F. Breitenecker and M. Graeff, Computing optimal controls for systems with state and control constraints, in: Proceedings of the IFAC Control Applications of Nonlinear Programming and Optimization, (1989), 39-44.

[17] H. Jaddu, Spectral method for constrained linear-quadratic optimal control. Math. Comput. in Simulation, 58 (2002), 159-169.

[18] M. Evrenosoglu and S. Somali, Least squares methods for solving singularity perturbed two-points boundary value problems using Bézier control point. Appl. Math. Letters, 21(10) (2008), 1029-1032.

[19] W. Rudin, Principles of mathematical analysis, McGraw-Hill (1986). 